\title{
5. THE IDENTIFICATION OF WHITE DWARF SUSPECTS IN THE LOWELL PROPER MOTION PROGRAM
}

\author{
H. L. GICLAS \\ Lowell Observatory, Flagstaff, Ariz., U.S.A.
}

During the course of the Lowell proper motion survey that has been in progress since 1957 , an effort has been made to identify the very blue stars and more recently the extremely red ones. The proper motions are detected and measured directly by projection on the calibrated grid of the blink microscope. The regular program catalogs all motions found $>0$."26/year and fainter than the eighth magnitude. From this portion of the program, we have identified 179 white dwarf suspects, 48 of color class - 1 and 131 of color class 0, which have been published in the Lowell Bulletins under the title of 'Lowell Proper Motions II through XV'. These lists cover the entire northern hemisphere, and a few regions down as far as $-10^{\circ}$ South, and contain 10382 different individual stars. Finding charts are provided for each object.

In order to realize a high degree of completeness at the lower adopted limit of motion, an attempt is made to mark all stars showing detectable motion; this includes many motions as small as $0.10 / y$ r. If, when comparing a blue with a red plate for color estimates, any of these marked stars or any others in the vicinity that show no appreciable motion appear to be very blue, they are noted. It is from these candidates that the 'GD' lists of 'White Dwarf Suspects' are prepared. Three such lists have been published (Lowell Bulletin, Nos. 125, 141, and 153) and contain 562 white dwarf suspects of various degrees of certainty. The most recent list (Lowell Bulletin, No. 153) of $155 \mathrm{GD}$ stars has just been completed as a part of this report, and completes the list of white dwarf suspects of small motions from $+50^{\circ}$ to the north pole.

In order to assess the true number of new white dwarfs contained in these lists, we refer to the papers of Eggen and Greenstein [3, 4, 5, and 6] where, either from the photometry by Eggen or from spectra taken by Greenstein, they have found that essentially all stars of color class -1 and $60 \%$ of color class 0 are white dwarfs (7). Therefore, one may expect to verify 145 white dwarfs from the regular program, and 335 from the GD lists for a total of 480 new ones. This does not include several hundred additional ones that might have Luyten, Tonanzintla, or other previous references that are included in both lists.

The number of stars in each color and motion class for the GD stars is shown in Figure 1. Most are in color class 0, and about equally distributed between our EPM (Estimated Proper Motion) 1 and 2, the small motions.

Plotting measured small proper motions from several sources, mostly Luyten, against our EPM, we find the relation shown in Figure 2. These are compared in Table I with the values used by Eggen and Greenstein [5], denoted EG in the table, to check the luminosity classes from the tangential motion. They differ slightly, but are based on perhaps twice as many small motions as were available to them. 


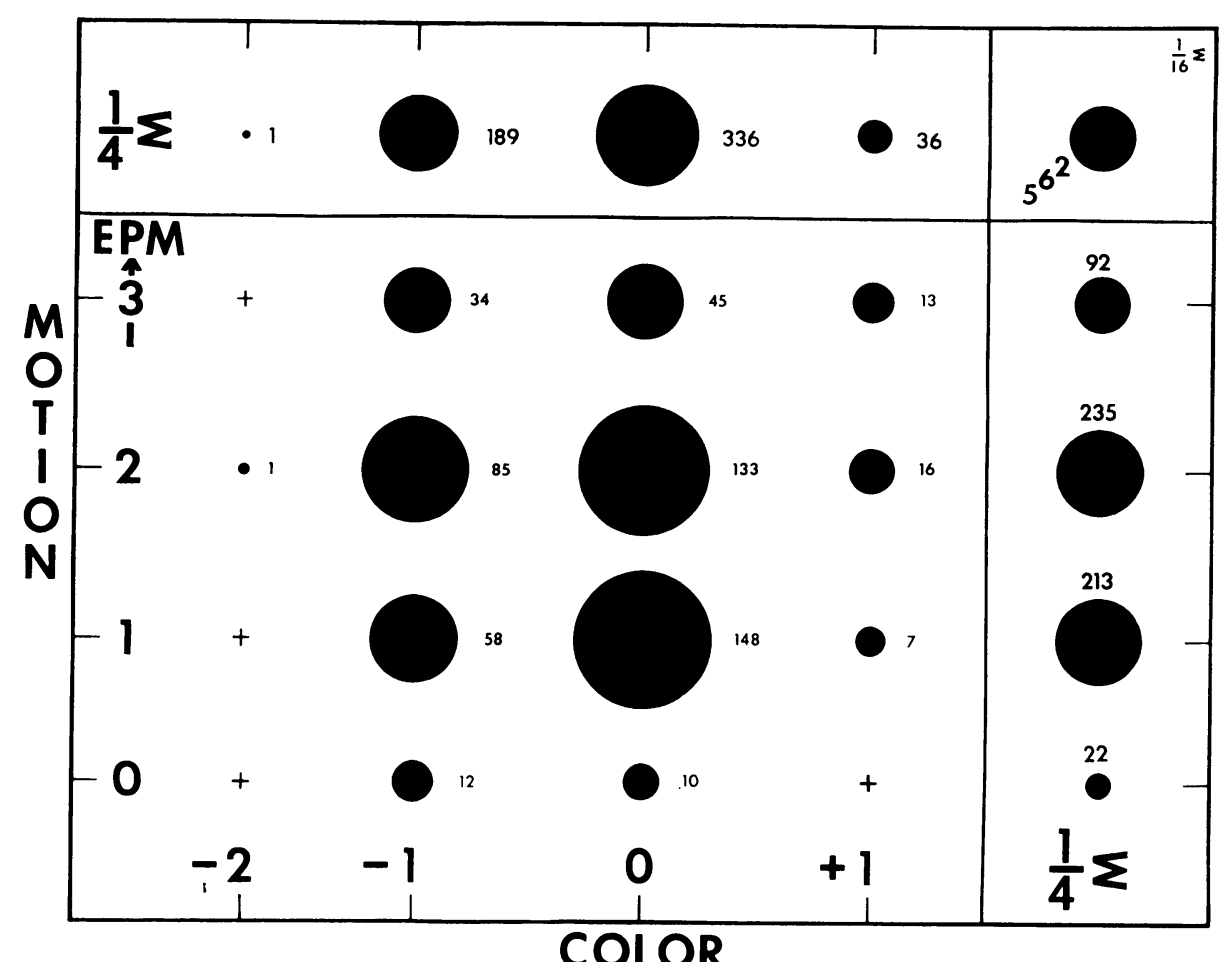

Fig. 1. The number of GD Stars in each color and EPM (Estimated Proper Motion) class.

To place a quantitative value on our color classes for the GD suspects is a little more involved if we want to define a reasonable narrow range. If we plot the UBV color of confirmed white dwarfs against our color classes 0 and -1 , we find the distribution shown in Figure 3. The mean value for the color class taken from this figure is shown in Table II. Examination of the $\mathrm{U}-\mathrm{B}$ range of colors for the 0 class stars in Figure 3 shows a marked secondary distribution around $U-B=-0.14$ mag. This group of objects was identified as stars with the smallest proper motion.

\section{TABLE I}

\begin{tabular}{|c|c|c|c|}
\hline \multirow[t]{2}{*}{ Lowell class } & & \multirow{2}{*}{$\begin{array}{l}\text { Average values } \\
\text { Motion }\end{array}$} & \\
\hline & & & Deviation \\
\hline EPM 3 & $\begin{array}{l}\mathrm{L}= \\
\mathrm{EG}=\end{array}$ & $\begin{array}{l}0^{\prime \prime} .213 \\
0^{\prime \prime} .246\end{array}$ & $\begin{array}{l} \pm 0^{\prime \prime} .037 \\
\pm 0^{\prime \prime} .049\end{array}$ \\
\hline EPM 2 & $\begin{array}{l}\mathrm{L}= \\
\mathrm{EG}=\end{array}$ & $\begin{array}{l}0^{\prime \prime} .168 \\
0^{\prime \prime} .167\end{array}$ & $\begin{array}{l} \pm 0^{\prime \prime} .036 \\
\pm 0^{\prime \prime} .034\end{array}$ \\
\hline EPM 1 & $\begin{array}{l}\mathrm{L}= \\
\mathrm{EG}=-\end{array}$ & $\begin{array}{l}0^{\prime \prime} .107 \\
0^{\prime \prime} .112\end{array}$ & $\begin{array}{l} \pm 0^{\prime \prime} .026 \\
\pm 0^{\prime \prime} .025\end{array}$ \\
\hline
\end{tabular}


As evidenced by the greater range of color for 0 class dwarf suspects, one would expect to find a more diverse assortment of objects to be included in that color class. To see if there may be a sorting of different types of degenerate stars dependent upon apparent tangential velocity, the B-V and $U-B$ has been plotted for each EPM. This is shown in Figures 4 and 5, and the color equivalents derived from them shown

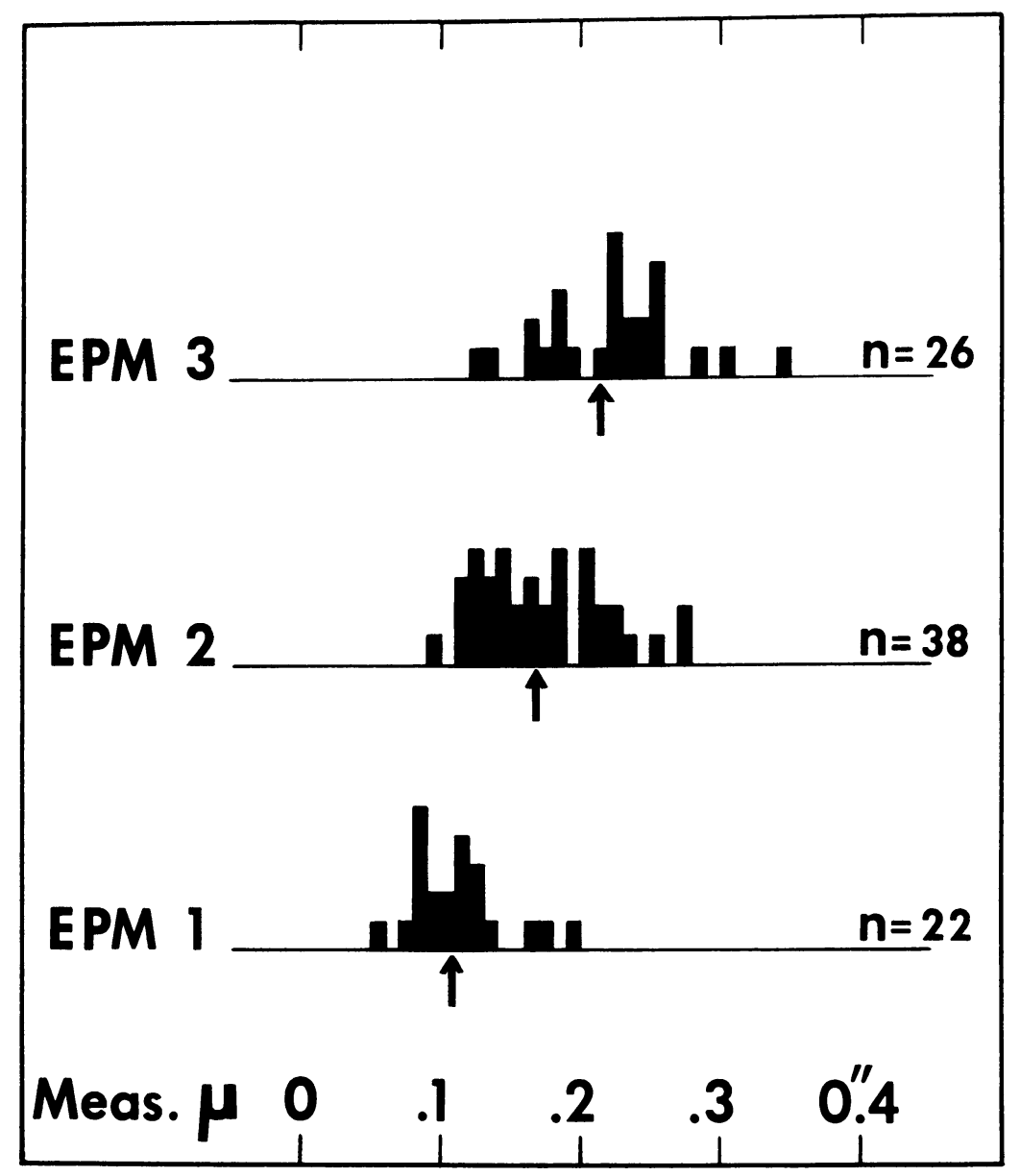

Fig. 2. Estimated Proper Motion, EPM, vs measured motion of Luyten and others.

in the last three columns of Table II. A dual value for the U-B of 0 color and EPM 1 class is given; one based on all stars, $U-B=-0.60$, and one, $U-B=-0.14$, indicated by an asterisk in Table I, for the subdwarfs. It is apparent from this that a preponderance of subdwarfs have been identified among the 0 color class with the smallest proper motion.

Greenstein [6], out of 86 GD stars classified spectroscopically, found 54 were early 
type white dwarfs, and the greatest difference between them and the program stars with large proper motion is the higher frequency of $\mathrm{DB}$, or helium rich stars. Thirtytwo of this sample were not white dwarfs; 22 of the 32 were horizontal branch stars or yellow subdwarfs that appear blue on our plates because of their large ultra-

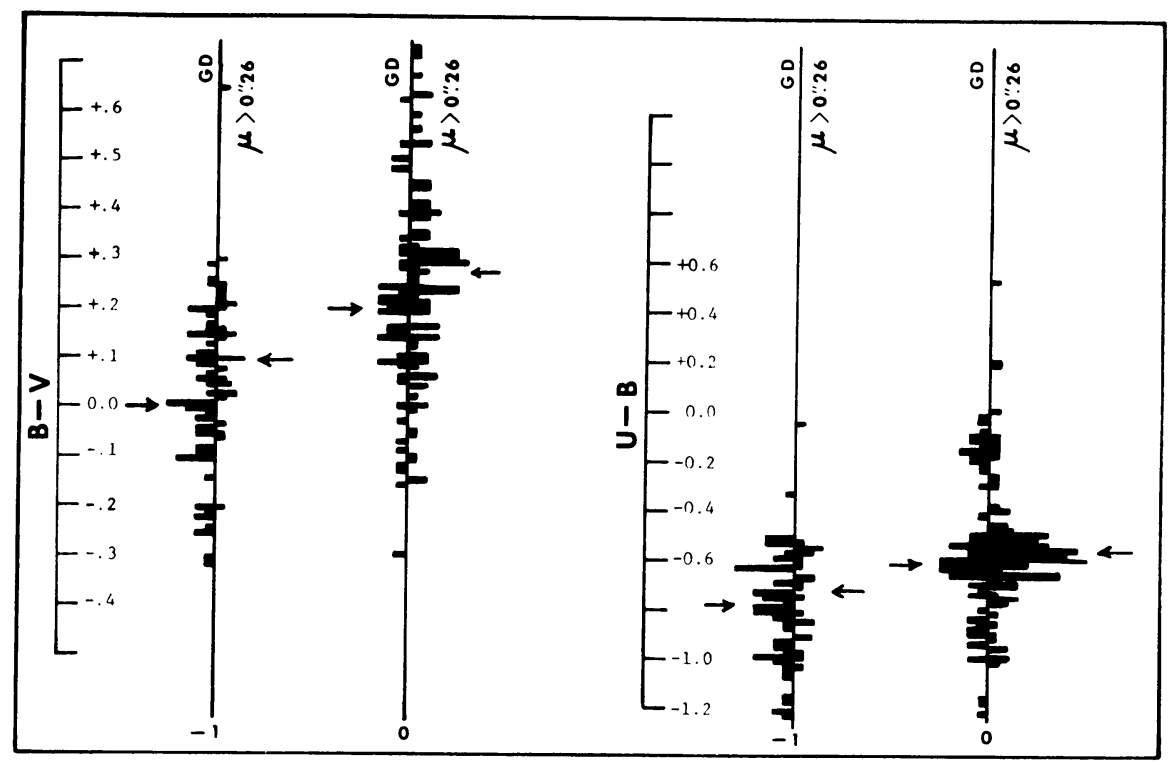

Fig. 3. B $-V$ and $U-B$ vs Lowell color class 0 and -1 for the confirmed GD and $\mu>0$ ". $26 / y$ r stars.

TABLE II

Figure 3

\begin{tabular}{rrrr} 
& $C$ & GD & $\mu>0^{\prime \prime} .26$ \\
& & & \\
\hline B $-\mathrm{V}$ & -1 & 0.00 & +0.10 \\
& 0 & +0.20 & +0.28 \\
U - B & -1 & -0.78 & -0.72 \\
& 0 & -0.60 & -0.55
\end{tabular}

Figure 4 and 5

EPM

12

2

3

0.00
+0.35
-0.78
-0.60

$-0.14^{*}$

$\begin{array}{rr}0.00 & +0.13 \\ +0.22 & +0.18 \\ -0.78 & -0.65 \\ -0.55 & -0.65\end{array}$

violet excess. A detailed discussion of the GD stars can be found in Greenstein's paper, 'The Lowell Suspect White Dwarfs' [6].

While the value for the position angle of the GD's is admittedly very rough, being estimated to the nearest $5^{\circ}$, an attempt to detect any excess number of these small motion objects moving toward selected convergents was investigated. The number of these stars moving toward each of the following three convergents were identified by 
the residual angle [9] technique previously described:

$\begin{array}{lrr}\text { Hyades } & 6^{h} 08^{\mathrm{m}} & +9^{\circ} 00^{\prime} \\ \text { 61 Cygni } & 637 & +030 \\ \text { Ursa Major } & 2021 & -5400\end{array}$

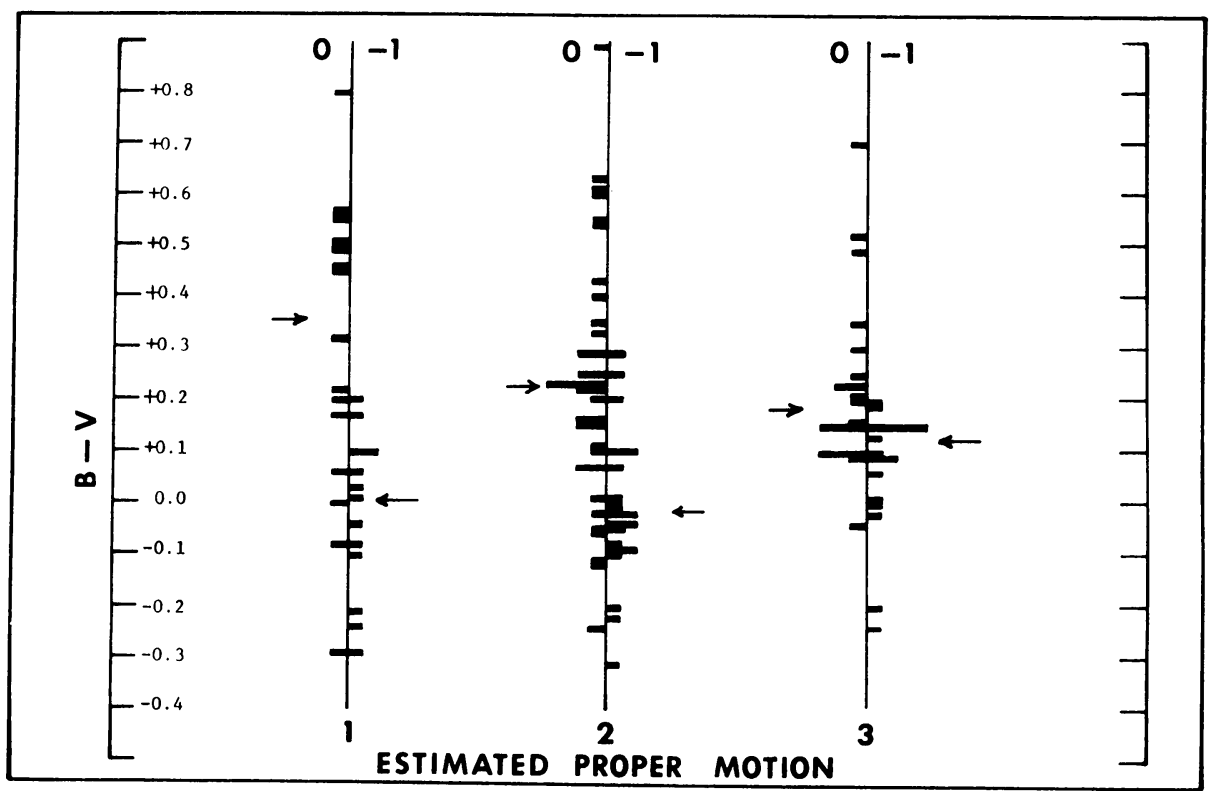

Fig. 4. B - V vs EPM for the Lowell GD dwarfs of color class 0 and -1 .

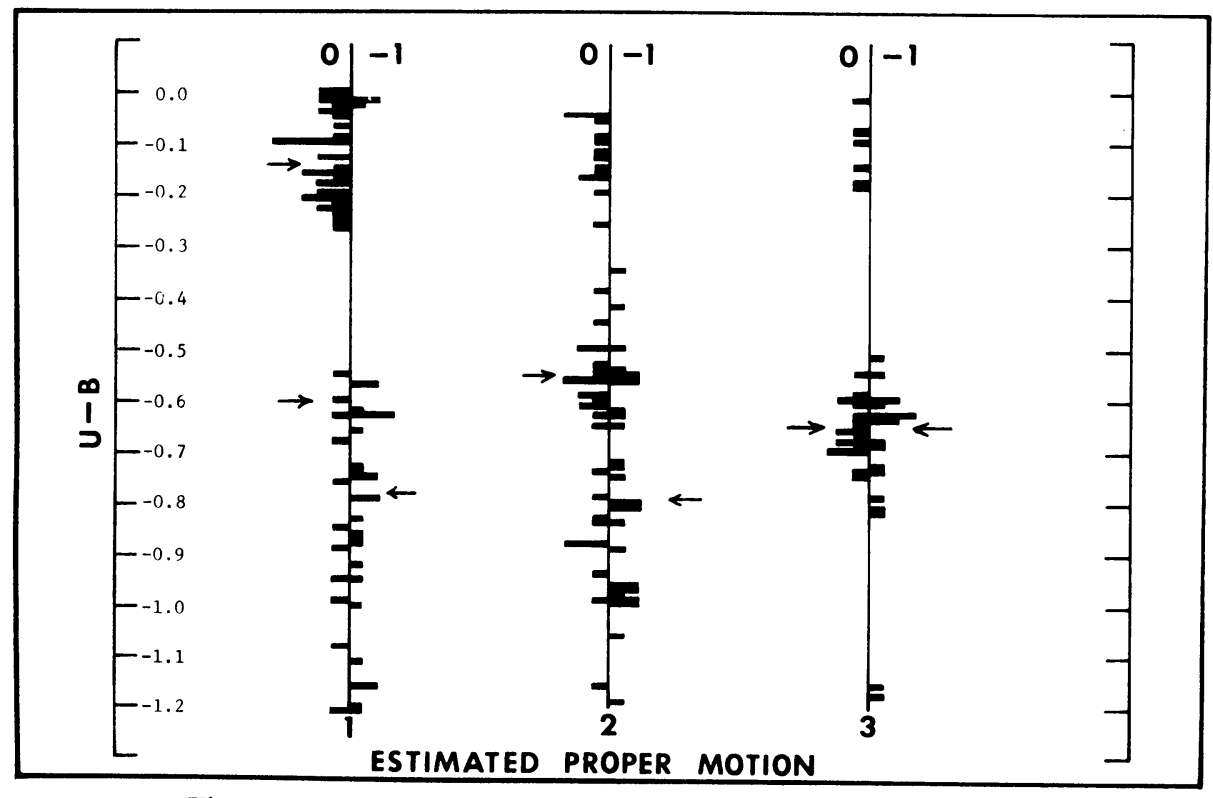

Fig. 5. $\mathbf{U}-\mathrm{B}$ vs EPM for the GD dwarfs of color class 0 and -1 . 
This procedure maximizes the separation of motion toward a convergent from the nearby Kapteyn Drift.

It was only in the case of the Hyades Group that there appeared to be an excess number of stars moving toward a convergent. The plot of the distribution of the residual angles is shown in Figure 6, where the Kapteyn Drift I has been taken to be $7^{h} 09^{\mathrm{m}}-13^{\circ} 00^{\prime}$, the position of the weighted direction of streaming determined from our own data [9]. The position of the Hyades convergent is at $0^{\circ}$, abscissa, of the figure, and the number of stars moving in that direction is the ordinate. The normal number of background stars within $40^{\circ}$ of the direction of the Hyades convergent, determined by symmetry of the curve around Drift I, should be 21 stars. Actually, the total number of stars found under the secondary, dotted, hump is 32 , therefore there appears to be an excess number of 11 stars travelling towards this convergent point. These 32 stars are listed in Table III. UBV photometry has been done by Eggen [10] for 15 of these, and are identified by the E10 appearing in the column headed References. Accurate proper motion and radial velocities will be needed to verify which ones of this list might be true Hyades Group members.

The Lowell proper motion program has been completed in the northern hemisphere, and the data is being prepared to print out a catalog ordered in right ascension, after averaging duplicate measures from overlapping plates. Columns will be provided for UBV photometry and reference to parallaxes where they exist. It is planned to continue the program to at least $-30^{\circ}$ South declination as first epoch plates taken

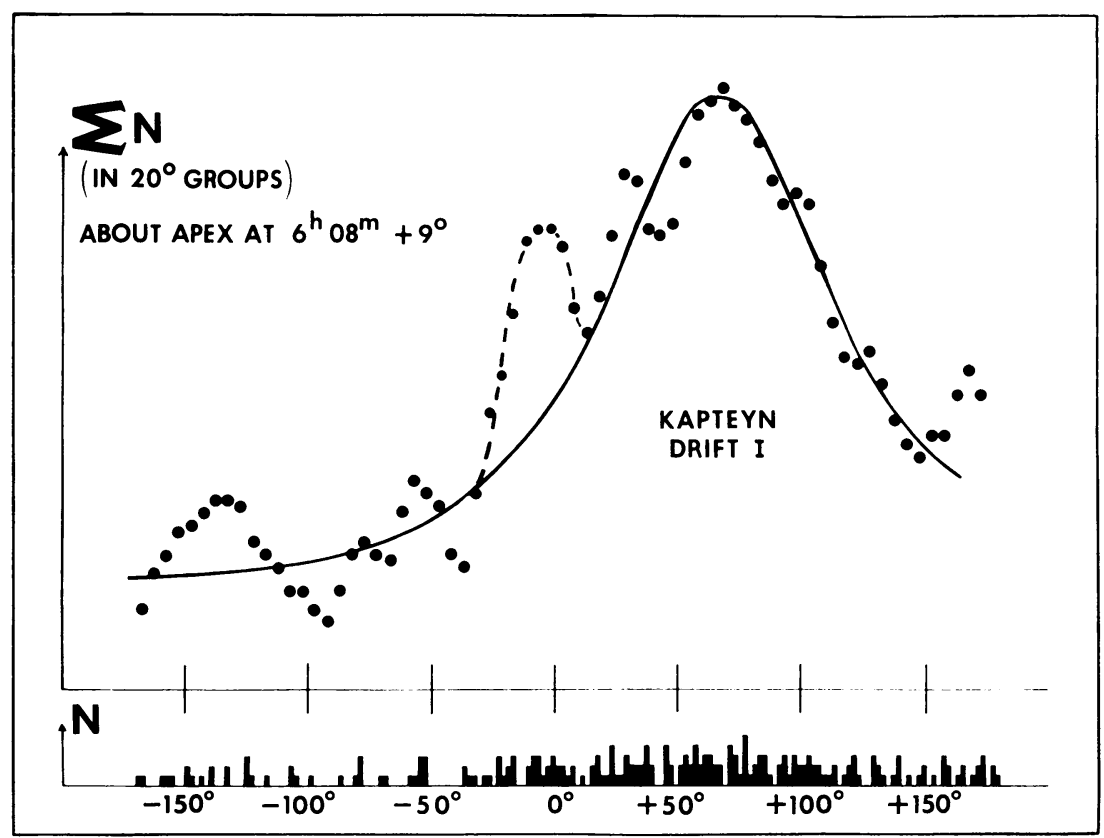

Fig. 6. Distribution of residual angle. Abscissa, position angle from Hyades group Apex at $0^{\circ}$, ordinate, number of stars. 
with the 13-inch telescope are available to declination $-40^{\circ}$. We are now in the process of moving this telescope to a dark sky site 12 miles $\mathrm{SE}$ of its present city location so that second epoch plates can be made to match the sky background of the first.

Some supplementary things we would like to do are to re-examine the first 60 -plate

TABLE III

List containing eleven possible Hyades group members

\begin{tabular}{|c|c|c|c|c|c|c|c|}
\hline Number & R.A. & Dec. & EPM & P.A. & Mag. & Color & References \\
\hline GD 6 & $0^{\mathrm{h}} 28^{\mathrm{m}} 07^{\mathrm{s}}$ & $+16^{\circ} 44^{\prime}$ & 1 & $80^{\circ}$ & 16.0 & +1 & \\
\hline 9 & $\begin{array}{ll}58 & 29\end{array}$ & -427 & 1 & 80 & 15.0 & -1 & PHL 940, E10 \\
\hline 19 & 15026 & +934 & 2 & 80 & 14.0 & 0 & E10 \\
\hline 31 & $\begin{array}{lll}2 & 31 & 37\end{array}$ & -524 & 3 & 70 & 14.0 & 0 & PHL 1358, E10 \\
\hline 51 & $347 \quad 54$ & -1344 & 2 & 60 & 15.0 & 0 & E10 \\
\hline 52 & $348 \quad 48$ & +3358 & 2 & 120 & 15.0 & -1 & E10 \\
\hline 436 & $\begin{array}{lll}5 & 17 & 39\end{array}$ & +6025 & 1 & 165 & 15.5 & 0 & \\
\hline 437 & 1859 & +7137 & 2 & 170 & 13.5 & 0 & LP33-137 \\
\hline 440 & $44 \quad 34$ & +7615 & 2 & 175 & 14.5 & 0 & \\
\hline 75 & $\begin{array}{lll}628 & 38\end{array}$ & +4739 & 3 & 190 & 14.0 & 0 & E10 \\
\hline 77 & 63726 & +4747 & 3 & 190 & 15.0 & -1 & E10 \\
\hline 292 & $41 \quad 26$ & +5335 & 1 & 195 & 15.0 & 0 & \\
\hline 84 & $\begin{array}{lll}7 & 14 & 23\end{array}$ & +4553 & 3 & 205 & 15.5 & -1 & E10 \\
\hline 111 & 100246 & +4302 & 1 & 260 & 16.0 & -1 & E10 \\
\hline 463 & $16 \quad 13$ & +6308 & 1 & 250 & 13.5 & 0 & \\
\hline 464 & 2326 & +6802 & 1 & 255 & 16.5 & +1 & \\
\hline 125 & 5201 & +2722 & 2 & 270 & 14.0 & -1 & Ton $556, \mathrm{E} 10$ \\
\hline 466 & $\begin{array}{lll}11 & 02 \quad 25\end{array}$ & +7452 & 2 & 255 & 15.0 & 0 & \\
\hline 127 & $02 \quad 42$ & +032 & 3 & 280 & 16.0 & 0 & E10 \\
\hline 135 & 1702 & -222 & 3 & 280 & 15.0 & 0 & E10 \\
\hline 308 & $22 \quad 59$ & +4240 & 2 & 270 & 16.0 & -1 & \\
\hline 146 & $12 \quad 00 \quad 34$ & +2118 & 2 & 280 & 16.0 & 0 & E10 \\
\hline 164 & $14 \quad 13 \quad 38$ & +2821 & 1 & 300 & 16.0 & 0 & E10 \\
\hline 343 & $1519 \quad 00$ & +6340 & 2 & 320 & 16.0 & -1 & LP68-60 \\
\hline 187 & $38 \quad 36$ & +3318 & 3 & 320 & 16.0 & 0 & LTT 14655 \\
\hline 194 & $50 \quad 12$ & +1819 & 2 & 310 & 14.5 & 0 & LTT 14705, E10 \\
\hline 359 & 170605 & +3536 & 2 & 340 & 16.0 & --1 & \\
\hline 364 & $\begin{array}{ll}45 & 13\end{array}$ & +3953 & 1 & 360 & 16.5 & -1 & \\
\hline 396 & $2143 \quad 16$ & +3518 & 3 & 60 & 15.5 & -1 & \\
\hline 240 & $2240 \quad 09$ & -430 & 3 & 80 & 16.0 & -1 & PHL 380, Feige 106 \\
\hline 562 & $2350 \quad 41$ & +7437 & 1 & 85 & 15.0 & 0 & \\
\hline 255 & $\begin{array}{ll}58 & 36\end{array}$ & +2038 & 2 & 80 & 15.5 & +1 & \\
\hline
\end{tabular}

regions for white dwarf suspects, and for extremely red stars. In continuing the program into the southern hemisphere it will be possible to match first epoch plates around the south galactic pole made in 1934, and to do a proper motion study in that area in depth. Perhaps the most useful addition to our program would be to do the UBV photometry of the dwarf suspects and other interesting objects; however, additional support would have to be found for this. 


\section{Acknowledgments}

It is a pleasure to acknowledge the contributions of my colleagues, Mr. Robert Burnham, Jr. and Mr. Norman G. Thomas, who have been with the Lowell proper motion program for the past 12 and 11 years respectively. This program has been supported by National Science Foundation grants which are gratefully acknowledged.

\section{References}

[1] Giclas, H. L., Burnham, Jr., R., and Thomas, N. G.: 1957-1970, 'Lowell Proper Motions' I through XV; Lowell Observatory Bulletin, Nos. 89, 102, 112, 120, 122, 124, 129, 132, 136, 138, $140,144,150,151$ and 152.

[2] Giclas, H. L., Burnham, Jr., R., and Thomas, N. G.: 'A List of White Dwarf Suspects', I, II and III; Lowell Observatory Bulletin. Nos. 125, 1965; 141, 1967 and 151, 1970.

[3] Eggen, O. J. and Greenstein, J. L.: 1965a, Astrophys. J. 141, 83.

[4] Eggen, O. J. and Greenstein, J. L.: 1965b, Astrophys. J. 142, 925.

[5] Eggen, O. J. and Greenstein, J. L.: 1967, Astrophys. J. 150, 927.

[6] Greenstein, J. L.: 1969, Astrophys. J. 158, 281.

[7] Annual Report of the Director 1964-1965, Mt. Wilson and Palomar Observatories.

[8] Annual Report of the Director 1965-1966, Mt. Wilson and Palomar Observatories.

[9] Giclas, H. L.: 1969, Low Luminosity Stars, Kumar, p. 28.

[10] Eggen, O. J.: 1968, Astrophys. J., Suppl. Series 16, 97, No. 143. 